\title{
Quantum entanglement and the Bell matrix
}

\author{
Anna Chiara Lai ${ }^{1}$ - Marco Pedicini' ${ }^{1}$ (D) \\ Silvia Rognone ${ }^{1}$
}

Received: 18 September 2015 / Accepted: 8 March 2016 / Published online: 13 April 2016

(C) Springer Science+Business Media New York 2016

\begin{abstract}
We present a class of maximally entangled states generated by a highdimensional generalisation of the CNOT gate. The advantage of our constructive approach is the simple algebraic structure of both entangling operator and resulting entangled states. In order to show that the method can be applied to any dimension, we introduce new sufficient conditions for global and maximal entanglement with respect to Meyer and Wallach's measure.
\end{abstract}

Keywords Quantum entanglement · Entangled states · Multipartite entanglement · CNOT $\cdot$ Bell states

Mathematics Subject Classification $81 \mathrm{P} 68 \cdot 81 \mathrm{P} 40$

\section{Introduction}

Entanglement is a key feature of quantum mechanical systems with wide applications to the field of quantum information theory. The class of quantum processes relying on

Partially supported by PRIN2011 Project "Metodi Logici per il trattamento dell'informazione".

$凶$ Marco Pedicini

marco.pedicini@uniroma3.it

Anna Chiara Lai

aclai@mat.uniroma3.it

Silvia Rognone

rognone.silvia@gmail.com

1 Dipartimento di Matematica e Fisica, Università Roma Tre, Via della Vasca Navale 84, 00146 Rome, Italy 
entangled states include quantum state teleportation [2], quantum error correction [3], quantum cryptography [15], and some quantum computational speedups [9]. Multiqubit entangled states are regarded as a valuable resource for processing information: for instance, several authors applied multi-qubit entanglement (and related entangling procedures) to multi-agent generalizations of the quantum teleportation protocol introduced in the paper by Bennett et al. [2]—-see for instance [23]. Also, other classes of multi-qubit entangled states turned out to be suitable for superdense coding.

Applications to quantum information theory motivated the search for the mathematical characterisations of multi-particle entanglement and for highly entangled quantum states. The approaches to this problem include an analytical classifications of entangled states [4,22], numerical optimisation techniques [5], and geometric characterisations [13].

Here, we present an orthonormal base of maximally entangled states, that we call general Bell states or $2^{n}$-dimensional Bell states, generated by an arbitrarily highdimensional generalisation of the CNOT gate. The advantage of our approach is the simple algebraic structure of both entangling gates and resulting states. In order to show the full generality of the method, we prove new sufficient conditions for both global entanglement and maximal entanglement (with respect to Meyer and Wallach's measure, see Eq.(1)): being based on the expectation value of an explicitly given operator, these criteria feature a simple formulation, scalability and observability.

In [17] Osterloh and Siewert propose a general method to construct new classes of entanglement measures based on suitable products and combinations of Pauli's matrices. Inspired by this approach, as well as by the multi-qubit concurrence proposed in [1] and by the relation between antilinear operators and concurrence [21], in what follows, we introduce a particular antilinear operator (Definition 4) and we use its expectation value as an entanglement criterion (Proposition 6) for general Bell states. In Proposition 9, we show that such an operator turns out to be related to Meyer and Wallach's (MW) measure [16] and we employ this relation to show that the general Bell states are maximally entangled with respect to this measure-Theorem 17.

To the best of our knowledge, an univoque and commonly accepted notion of entanglement measure in high-dimensional systems has not yet been introduced. Several proposals in the literature try to capture distinct aspects of a maximally entangled state. For instance, the Schmidt decomposition, see [8], induces a measure related to the minimum number of terms in the product expansion of a state, while the fully entangled fraction measures the ability of a state to perform tasks related to quantum computing, such as teleportation and dense coding [12].

Brown et al. and Tapiador et al. [7,20] used a computationally tractable entanglement measure based on the negative partial transpose criterion. Another relevant attempt, introduced in $[10,14]$, was to define the maximal entanglement through four equivalent criteria; moreover, by following this approach, maximally entangled states determine an orthogonal basis.

Throughout this paper, we focus on MW measure [16]. This measure interprets the global entanglement as the average bipartite entanglement of every qubit with respect to the rest of the system. It has thus the advantage of a simple physical meaning as well as a simple formulation, introduced in [6]: 


$$
Q(|\psi\rangle):=2\left(1-\frac{1}{n} \sum_{j=1}^{n} \operatorname{Tr}\left[\rho_{j}^{2}\right]\right)
$$

where $n$ is the number of qubits of the system, $\rho_{j, \psi}$ is the density matrix obtained by tracing out the $j$-th qubit of the state $|\psi\rangle$ and $\operatorname{Tr}[\cdot]$ represents the trace operator.

The main result we present here is a sufficient condition on multi-qubit states to be maximally entangled (with respect to MW measure) and, as mentioned above, we establish this result in order to show that a set of states generalising Bell states have maximal MW measure.

The paper is organised as follows. In Sect. 2 we show sufficient conditions for global entanglement and for the maximality of the MW measure of a state in a multi-qubit system. In Sect. 3 we propose a generalisation of the CNOT gate to multi-qubit systems a related class of states, that we call $2^{n}$-dimensional Bell states. By applying the criteria introduced in Sect.2, we are able to show that these generalisations of Bell states are maximally entangled with respect to MW measure. Some possible extensions of this approach are illustrated in Sect. 3.1.

\section{An entanglement criterion}

First of all we give the formal definition of globally entangled state.

Definition 1 A state $|\psi\rangle$ is globally entangled if for any $\left|\phi_{1}\right\rangle$ and $\left|\phi_{2}\right\rangle$ we have $|\psi\rangle \neq$ $\left|\phi_{1}\right\rangle \otimes\left|\phi_{2}\right\rangle$.

Remark 2 Throughout this paper we consider elements of Hilbert spaces $|\psi\rangle \in \mathbb{C}^{2^{n}}$ which are pure quantum states, i.e., they are complex vectors of unit Euclidean norm: $|\psi\rangle=\left(\psi_{1}, \ldots, \psi_{2^{n}}\right)$ and $\sum_{j=1}^{2^{n}}\left|\psi_{j}\right|^{2}=1$; for brevity we refer to them simply as "states".

Notation 3 We use the symbol $I_{2^{n}}$ to denote the $2^{n}$-dimensional identity matrix:

$$
I_{2^{n}}:=\underbrace{I_{2} \otimes \cdots \otimes I_{2}}_{n-\text { times }} .
$$

being $I_{2^{n}}=(1)$ if $n=0$.

The expectation value of the operator $A$ in the state $\psi$ is denoted by

$$
\langle A\rangle_{\psi}:=\langle\psi|A| \psi\rangle
$$

Moreover we denote by $\sigma_{y}$ the Pauli matrix

$$
\left(\begin{array}{cc}
0 & -i \\
i & 0
\end{array}\right)
$$


We introduce the following two operators, they are used to define the particular antilinear operator we apply to states constructed with algorithm in Sect. 3 in order to prove they are entangled states.

Definition 4 Let us denote by $\mathcal{F}: \mathbb{C}^{2^{n}} \rightarrow \mathbb{C}$ the function which associates to a state $|\psi\rangle$ the expectation value of the operator $M_{2^{n}} K_{2^{n}}$ in the state $|\psi\rangle$, namely:

$$
\mathcal{F}(|\psi\rangle):=\left\langle M_{2^{n}} K_{2^{n}}\right\rangle_{\psi}
$$

where $M_{2^{n}}:=\sigma_{y} \otimes I_{2^{n-2}} \otimes \sigma_{y}$ and $K_{2^{n}}$ is the conjugation operator.

Note that

$$
\mathcal{F}(|\psi\rangle):=\left\langle M_{2^{n}} K_{2^{n}}\right\rangle_{\psi}=\left\langle\psi\left|M_{2^{n}} K_{2^{n}}\right| \psi\right\rangle=\left\langle\psi\left|M_{2^{n}}\right| \bar{\psi}\right\rangle
$$

where $|\bar{\psi}\rangle$ denotes the complex conjugate of $|\psi\rangle$.

Example 5 We explicitly compute $M_{4}$ :

$$
\begin{aligned}
M_{4} & :=\sigma_{y} \otimes I_{2^{2-2}} \otimes \sigma_{y}=\sigma_{y} \otimes(1) \otimes \sigma_{y} \\
& =\sigma_{y} \otimes \sigma_{y}=\left(\begin{array}{cc}
0 & -i \\
i & 0
\end{array}\right) \otimes \sigma_{y}=\left(\begin{array}{cc}
0 & -i \sigma_{y} \\
i \sigma_{y} & 0
\end{array}\right)=\left(\begin{array}{cccc}
0 & 0 & 0 & -1 \\
0 & 0 & 1 & 0 \\
0 & 1 & 0 & 0 \\
-1 & 0 & 0 & 0
\end{array}\right)
\end{aligned}
$$

For a representation of matrices $M_{2^{n}}$ with $n \geq 2$ see Fig. 1 .

We now show that $M_{2^{n}} K_{2^{n}}$ has zero expectation value on product states.

Proposition 6 If $|\psi\rangle$ is an unentangled state then $\mathcal{F}(|\psi\rangle)=0$.

Proof Let $n \geq 1,|\psi\rangle \in \mathbb{C}^{2^{n}},\left|\phi_{1}\right\rangle \in \mathbb{C}^{2^{n_{1}}},\left|\phi_{2}\right\rangle \in \mathbb{C}^{2^{n_{2}}}, n_{1}+n_{2}=n$ and assume $|\psi\rangle=\left|\phi_{1}\right\rangle \otimes\left|\phi_{2}\right\rangle$. Also let $\left|\bar{\phi}_{1}\right\rangle=(a, b) \in \mathbb{C}^{2^{n_{1}}}$ defined by $a, b \in \mathbb{C}^{2^{n_{1}-1}}$, two half vectors of its coordinates in the standard basis. One has

$$
\left(\sigma_{y} \otimes I_{2^{n_{1}-1}}\right)(a, b)=-i(b,-a)
$$

\section{Consequently}

$$
\left\langle\phi_{1}\left|\sigma_{y} \otimes I_{2^{n_{1}-1}}\right| \bar{\phi}_{1}\right\rangle=(a, b)^{\mathrm{T}}\left(\sigma_{y} \otimes I_{2^{n_{1}-1}}\right)(a, b)=-i a \cdot b+i b \cdot a=0 .
$$

Similarly, if $\left|\bar{\phi}_{2}\right\rangle=\left(y_{1}, \ldots, y_{2^{n_{2}}}\right)$ then

$$
\begin{aligned}
\left(I_{n_{2}-1} \otimes \sigma_{y}\right)\left|\bar{\phi}_{2}\right\rangle & =\left(I_{n_{2}-1} \otimes \sigma_{y}\right)\left(y_{1}, \ldots, y_{2^{n}}\right) \\
& =-i\left(y_{2},-y_{1}, y_{3},-y_{4}, \ldots, y_{2^{n_{2}}},-y_{2^{n_{2}-1}}\right)
\end{aligned}
$$



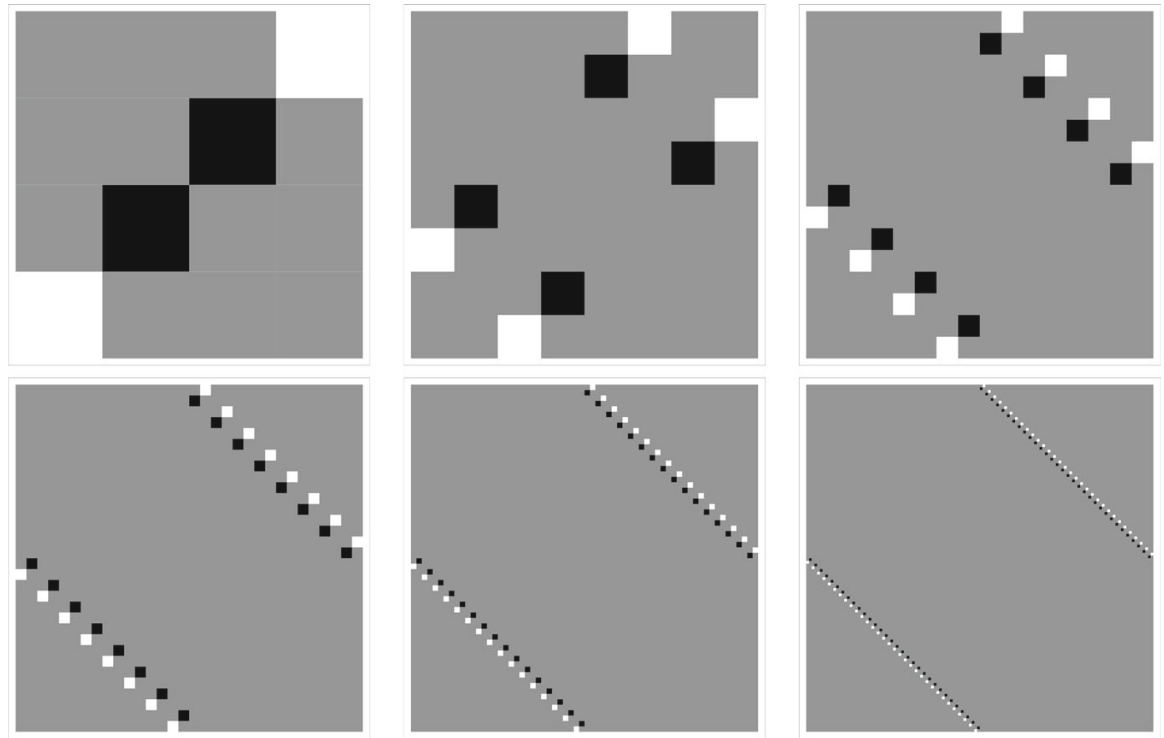

Fig. 1 We show in this picture the matrices $M_{2}$ for $n=2, \ldots, 7$. For each $n$, a matrix is represented as a square of fixed length, partitioned into $2^{n} \times 2^{n}$ pixels. Each pixel represents an entry of the matrix: for instance, the leftmost top pixel is related to the entry $M_{2^{n}}(1,1)$ of the corresponding matrix. The value of the entry is encoded by the colour of the corresponding pixel: entries 0 are shown in grey colour, entries 1 by black colour and entries -1 by white colour. For instance $M_{2^{n}}(1,1)=0$ for each $n=2, \ldots, 7$ and this information can be read in the picture by noting that the lefmost top pixel is always grey

thus

$$
\begin{aligned}
\left\langle\phi_{2}\left|I_{n_{2}-1} \otimes \sigma_{y}\right| \bar{\phi}_{2}\right\rangle & =\left(y_{1}, \ldots, y_{2^{n_{2}}}\right)^{\mathrm{T}}\left(I_{n_{2}-1} \otimes \sigma_{y}\right)\left(y_{1}, \ldots, y_{2^{n_{2}}}\right) \\
& =-i\left(y_{1}, \ldots, y_{2^{n_{2}}}\right)^{\mathrm{T}}\left(y_{2},-y_{1}, y_{3},-y_{4}, \ldots, y_{2^{n_{2}}},-y_{2^{n_{2}}-1}\right)=0 .
\end{aligned}
$$

By equalities (3) and (4) one finally has

$$
\begin{aligned}
\mathcal{F}(|\psi\rangle) & =\left\langle\psi\left|M_{2^{n}}\right| \bar{\psi}\right\rangle=\left\langle\phi_{1} \otimes \phi_{2}\left|\sigma_{y} \otimes I_{2^{n-2}} \otimes \sigma_{y}\right| \bar{\phi}_{1} \otimes \bar{\phi}_{2}\right\rangle \\
& =\left\langle\phi_{1} \otimes \phi_{2}\left|\sigma_{y} \otimes I_{2^{n_{1}-1}} \otimes I_{2^{n_{2}-1}} \otimes \sigma_{y}\right| \bar{\phi}_{1} \otimes \bar{\phi}_{2}\right\rangle \\
& =\left\langle\phi_{1}\left|\sigma_{y} \otimes I_{n_{1}-1}\right| \bar{\phi}_{1}\right\rangle\left\langle\phi_{2}\left|I_{n_{2}-1} \otimes \sigma_{y}\right| \bar{\phi}_{2}\right\rangle=0 .
\end{aligned}
$$

Next result shows that $\mathcal{F}$ also provides a sufficient condition for maximal entanglement. It is useful to recall the following:

Definition 7 (Schmidt decomposition) Let $n_{1}, n_{2} \in \mathbb{N}$ such that $n_{1}+n_{2}=n$ and let $A=\mathbb{C}^{2^{n_{1}}}$ and $B=\mathbb{C}^{2^{n_{2}}}$ so that $\mathbb{C}^{2^{n}}=A \otimes B$. Then any state $|\psi\rangle \in \mathbb{C}^{2^{n}}$ can be written in the form

$$
|\psi\rangle=\sum_{k=1}^{K} c_{k}\left|\phi_{k}^{A}\right\rangle \otimes\left|\phi_{k}^{B}\right\rangle
$$


where $K=\min \{\operatorname{dim}(A), \operatorname{dim}(B)\}=\min \left\{2^{n_{1}}, 2^{n_{2}}\right\}, c_{k} \geq 0$ and $\left\{\left|\phi_{k}^{A}\right\rangle\right\},\left\{\left|\phi_{k}^{B}\right\rangle\right\}$ are two orthonormal subsets of $A$ and $B$, respectively, see [18]. This decomposition takes the name of Schmidt decomposition ${ }^{1}$.

Remark 8 Consider the decomposition $\mathbb{C}^{2^{n}}=A \otimes B$ and let $\rho_{A, \psi}$ be the density operator of the state $|\psi\rangle$ on the subsystem $A$. Then the set of the positive eigenvalues of $\rho_{A, \psi}$ coincides with the set $\left\{c_{k}^{2} \mid c_{k}>0\right\}$ of positive squared coefficients of Schmidt decomposition of the state $|\psi\rangle$ with respect to the decomposition $\mathbb{C}^{2^{n}}=A \otimes B$ - see for instance [19]. As a consequence, $\operatorname{Tr}\left[\rho_{A, \psi}\right]=\sum_{k=1}^{K} c_{k}^{2}=1$ and $\operatorname{Tr}\left[\rho_{A, \psi}^{2}\right]=$ $\sum_{k=1}^{K} c_{k}^{4}$.

Proposition 9 If $\mid \mathcal{F}(|\psi\rangle) \mid=1$ then $|\psi\rangle$ is maximally entangled with respect to $M W$ measure.

Proof First of all we notice that

(a) If $|\phi\rangle \in \mathbb{C}^{2}$ then $\left\langle\phi\left|\sigma_{y}\right| \bar{\phi}\right\rangle=0$;

(b) If $\left\{\left|\phi_{1}\right\rangle,\left|\phi_{2}\right\rangle\right\}$ is an orthonormal base of $\mathbb{C}^{2}$ then $\left|\left\langle\phi_{1}\left|\sigma_{y}\right| \bar{\phi}_{2}\right\rangle\right|=1$ and

$$
\left\langle\phi_{1}\left|\sigma_{y}\right| \bar{\phi}_{2}\right\rangle=-\left\langle\phi_{2}\left|\sigma_{y}\right| \bar{\phi}_{1}\right\rangle
$$

(c) For all $\left|\xi_{1}\right\rangle,\left|\xi_{2}\right\rangle \in \mathbb{C}^{2^{n-1}}$ one has

$$
\left|\left\langle\xi_{1}\left|I_{2^{n-2}} \otimes \sigma_{y}\right| \bar{\xi}_{2}\right\rangle\right| \leq 1
$$

and

$$
\left\langle\xi_{1} \mid I_{2^{n-2}} \otimes \sigma_{y} \bar{\xi}_{2}\right\rangle=-\left\langle\xi_{2}\left|I_{2^{n-2}} \otimes \sigma_{y}\right| \bar{\xi}_{1}\right\rangle
$$

Also remark that the Schmidt decomposition of $|\psi\rangle$ with respect the decomposition that singles out a generic qubit of the system reads:

$$
|\psi\rangle=\sum_{k=1}^{2} c_{k}\left|\phi_{k}\right\rangle \otimes\left|\xi_{k}\right\rangle
$$

for some $c_{1}, c_{2} \geq 0$ such that $c_{1}^{2}+c_{2}^{2}=1$, some orthonormal base $\left\{\left|\phi_{1}\right\rangle,\left|\phi_{2}\right\rangle\right\}$ of $\mathbb{C}^{2}$ and some orthonormal subset $\left\{\left|\xi_{1}\right\rangle,\left|\xi_{2}\right\rangle\right\}$ of $\mathbb{C}^{2^{n-1}}$.

In view of (a)-(c), we then have

$$
\begin{aligned}
\mid \mathcal{F}(|\psi\rangle) \mid & =\left|\sum_{k, h=1}^{2} c_{k} c_{h}\left\langle\phi_{k}\left|\sigma_{y}\right| \phi_{h}\right\rangle\left\langle\xi_{k}\left|I_{2^{n-2}} \otimes \sigma_{y}\right| \bar{\xi}_{h}\right\rangle\right| \\
& =\left|2 c_{1} c_{2}\left\langle\xi_{1}\left|I_{2^{n-2}} \otimes \sigma_{y}\right| \bar{\xi}_{2}\right\rangle\right| \leq\left|2 c_{1} c_{2}\right|
\end{aligned}
$$

\footnotetext{
1 More generally, the Schmidt decomposition is well defined for pure states belonging to general Hilbert spaces $X$.
} 
On the other hand $\left|2 c_{1} c_{2}\right| \leq 1$ for all $c_{1}, c_{2} \in \mathbb{R}$ such that $c_{1}^{2}+c_{2}^{2}=1$, and the maximum $\left|2 c_{1} c_{2}\right|=1$ is attained at the points satisfying $c_{1}^{2}=c_{2}^{2}=1 / 2$ (Fig. 2). Therefore we may conclude that if $\mid \mathcal{F}(|\psi\rangle) \mid=1$ then $c_{1}^{2}=c_{2}^{2}=1 / 2$. Since this argument holds for any qubit, we have that

$$
\operatorname{Tr}\left[\rho_{j, \psi}^{2}\right]=c_{1}^{4}+c_{2}^{4}=\frac{1}{2} \text { for all } j=1, \ldots, n
$$

see also Remark 8. Consequently,

$$
Q(|\psi\rangle)=2\left(1-\frac{1}{n} \sum_{j=1}^{n} \operatorname{Tr}\left[\rho_{j, \psi}^{2}\right]\right)=1 .
$$

Above results relate the value of $\mid \mathcal{F}(|\psi\rangle) \mid$ to a measure of entanglement of the state $|\psi\rangle$. In particular if $\mid \mathcal{F}(|\psi\rangle) \mid$ is minimal, i.e., $\mid \mathcal{F}(|\psi\rangle) \mid=0$, then $|\psi\rangle$ is not entangled while if $\mid \mathcal{F}(|\psi\rangle) \mid$ is maximal, i.e., $\mid \mathcal{F}(|\psi\rangle) \mid=1$ then $|\psi\rangle$ is maximally entangled. However, the condition $\mid \mathcal{F}(|\psi\rangle) \mid=0$ (respectively $\mid \mathcal{F}(|\psi\rangle) \mid=1$ ) is a sufficient but not necessary condition to have $|\psi\rangle$ unentangled (resp. maximally entangled). Indeed, consider the Greenberger-Horne-Zeilinger state

$$
\left|G H Z_{n}\right\rangle:=\frac{1}{\sqrt{2}}\left(\left|0_{n}\right\rangle+\left|1_{n}\right\rangle\right) .
$$

For all $n \geq 2$, the state $\left|G H Z_{n}\right\rangle$ is globally entangled state and yet, for $n \geq 3$, $\mathcal{F}\left(\left|G H Z_{n}\right\rangle\right)=0$ : this implies that, in general, the inverse implication of Proposition 6 (that is, $\mathcal{F}(|\psi\rangle)=0$ implies $|\psi\rangle$ is unentangled) is not true. Furthermore, for all $n \geq 2$, the state $\left|G H Z_{n}\right\rangle$ is maximally entangled with respect to MW measure and $\mathcal{F}(|\psi\rangle) \neq 1$, thus also the inverse implication of Proposition 9 (that is, $\mathcal{F}(|\psi\rangle)=1$ implies $|\psi\rangle$ is maximally entangled) in general is not true.

Fig. 2 Bell Circuit:

entanglement of two elements of the canonical basis $|0\rangle$ and $|1\rangle$

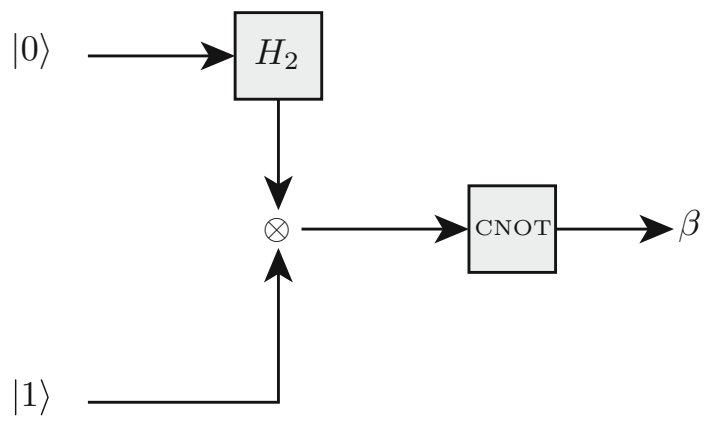




\section{$3 n$-qubit entanglement algorithm}

In this section we introduce a generalisation of the CNOT gate and we show that the resulting Bell states are fully entangled (Fig. 3).

To this end we adopt the following notations:

Notation 10 We use $H_{2}:=\frac{1}{\sqrt{2}}\left(\begin{array}{cc}1 & 1 \\ 1 & -1\end{array}\right)$ is the Hadamard matrix and

$$
H_{2^{n}}:=\underbrace{H_{2} \otimes \cdots \otimes H_{2}}_{n \text { times }}
$$

is its $2^{n}$-dimensional generalisation, i.e., the $2^{n}$-dimensional Walsh matrix. We use the symbols $\sigma_{x}, \sigma_{y}$ and $\sigma_{z}$ to denote Pauli's matrices

$$
\sigma_{x}=\left(\begin{array}{ll}
0 & 1 \\
1 & 0
\end{array}\right), \quad \sigma_{y}=\left(\begin{array}{cc}
0 & -i \\
i & 0
\end{array}\right), \quad \sigma_{z}=\left(\begin{array}{cc}
1 & 0 \\
0 & -1
\end{array}\right) .
$$

We finally consider the orthogonal projectors

$$
L:=\left(\begin{array}{ll}
1 & 0 \\
0 & 0
\end{array}\right), \quad R:=\left(\begin{array}{ll}
0 & 0 \\
0 & 1
\end{array}\right)
$$

In view of above notation, we remark that the CNOT gate satisfies the equality

$$
\text { CNOT }:=\left(\begin{array}{cccc}
1 & 0 & 0 & 0 \\
0 & 1 & 0 & 0 \\
0 & 0 & 0 & 1 \\
0 & 0 & 1 & 0
\end{array}\right)=L \otimes I_{2}+R \otimes \sigma_{x}
$$

while the columns of the matrix

$$
B_{4}:=\frac{1}{\sqrt{2}}\left(\begin{array}{cccc}
1 & 0 & 1 & 0 \\
0 & 1 & 0 & 1 \\
0 & 1 & 0 & -1 \\
1 & 0 & -1 & 0
\end{array}\right)=\operatorname{CNOT}\left(H_{2} \otimes I_{2}\right)
$$

are the coordinate vectors of the Bell states in the standard base. We extend the above definitions of CNOT and of $B_{2}$ to an arbitrary number of qubits as follows

Definition 11 For $n \geq 2$ we set

$$
\mathrm{CNOT}_{2^{n}}:=L \otimes I_{2^{n-1}}+R \otimes \underbrace{\sigma_{x} \otimes \cdots \otimes \sigma_{x}}_{n \text { times }}
$$

and

$$
B_{2^{n}}:=\mathrm{CNOT}_{2^{n}}\left(H_{2^{n-1}} \otimes I_{2}\right) .
$$



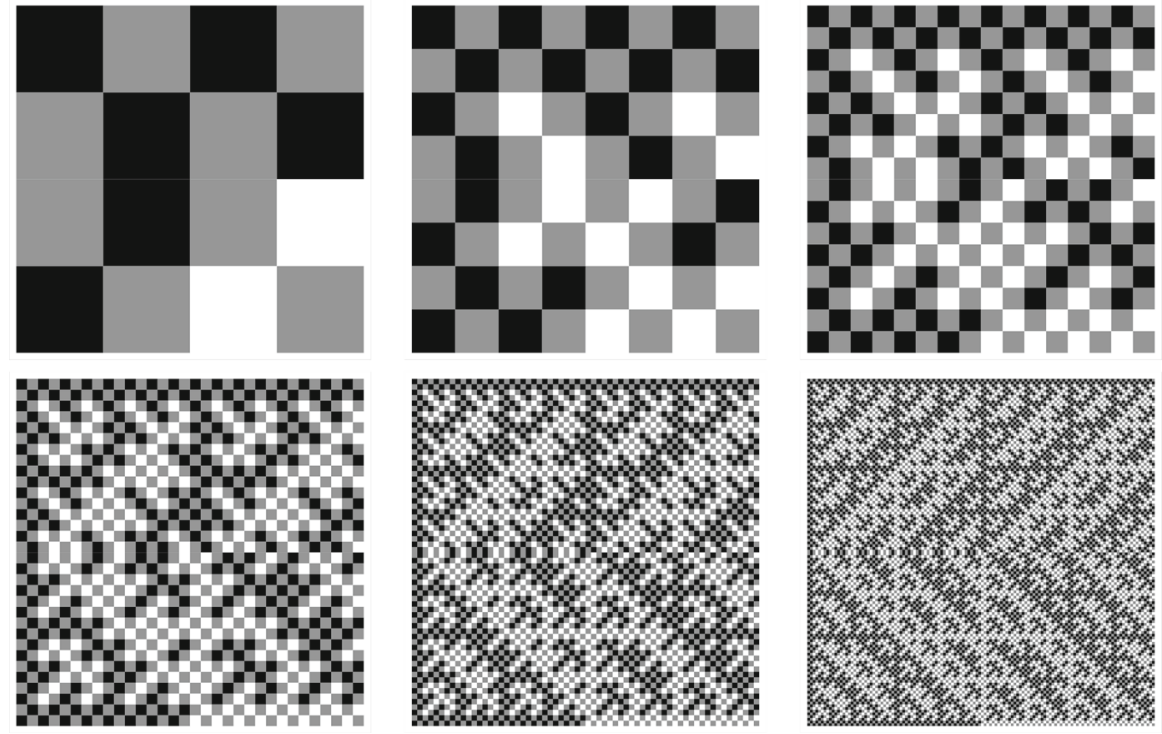

Fig. 3 A representation of Bell matrices $B_{2} n$ for $n=2, \ldots, 7$

We define $2^{n}$-dimensional Bell state any state

$$
\left|b_{k}\right\rangle:=B_{2^{n}}|k\rangle
$$

where $k=0, \ldots, 2^{n}-1$ and $|k\rangle$ is the $k$-th element of the standard base of $\mathbb{C}^{2^{n}}$.

Remark 12 Note that for any pair of square matrices $A$ and $B$ with same dimension, the matrix $L \otimes A+R \otimes B$ is usually referred to as their Kronecker sum; this operation shares with the Kronecker (tensor) product the property of preserving unitarity, [11, Chapter 8]. Then by construction, the matrix $B_{2^{n}}$ is product, tensor product and Kronecker sum of unitary matrices, and consequently, it is a unitary matrix. As columns of a unitary matrix, the Bell states $\left|b_{k}\right\rangle$, with $k=1, \ldots, 2^{n}$ form a complete orthonormal basis for $\mathbb{C}^{2^{n}}$

In what follows we show that the $2^{n}$-dimensional Bell states are maximally entangled with respect to MW measure. We introduce the matrix

$$
L_{2^{n}}:=B_{2^{n}}^{\dagger} M_{2^{n}} B_{2^{n}}
$$

whose relevance in our investigation is motivated by the following

Lemma 13 If $\left|\left\langle\phi\left|L_{2^{n}}\right| \bar{\phi}\right\rangle\right|=1$ and if $|\psi\rangle=B_{2^{n}}|\phi\rangle$ then $|\psi\rangle$ is maximally entangled with respect to the $M W$ measure.

In particular, if $\left|\left\langle k\left|L_{2^{n}}\right| \bar{k}\right\rangle\right|=1$, where $|k\rangle$ is the $k$-th element of the standard base, then the $k$-th Bell state is maximally entangled with respect to the MW measure. 
Proof By the definition of $L_{2^{n}}$ and by the assumption $|\psi\rangle=B_{2^{n}}|\phi\rangle$ one has

$$
\begin{aligned}
\left|\left\langle\phi\left|L_{2^{n}}\right| \bar{\phi}\right\rangle\right| & =\left|\left\langle\phi\left|B_{2^{n}}^{\dagger} M_{2^{n}} B_{2^{n}}\right| \bar{\phi}\right\rangle\right| \\
& =\left|\left\langle B_{2^{n}} \phi\left|M_{2^{n}}\right| \overline{\left(B_{2^{n}} \mid \phi\right)}\right\rangle\right|=\left|\left\langle\psi\left|M_{2^{n}}\right| \bar{\psi}\right)\right\rangle|=| \mathcal{F}(|\psi\rangle) \mid .
\end{aligned}
$$

The first part of the claim hence follows by Proposition 9.

The second part of the claim readily follows by applying above reasoning to $|\phi\rangle=$ $|k\rangle$ and by the definition of $2^{n}$-dimensional Bell state.

Remark 14 There exist states $\phi$ which not satisfy $\left|\left\langle\phi\left|L_{2^{n}}\right| \bar{\phi}\right\rangle\right|=1$ and such that $B_{2^{n}}|\phi\rangle$ is maximally entangled, an example of this phenomenon is given by the state $\phi=B_{2^{n}}^{-1}\left|G H Z_{n}\right\rangle$.

Next result gives a closed formula for $L_{2^{n}}$ and relates its diagonal elements to the Thue-Morse sequence, that is the binary sequence $\left(\tau_{i}\right)$ defined by the recursive relation

$$
\begin{aligned}
\tau_{1} & :=0 \\
\tau_{2 n}: & =1-\tau_{n} \\
\tau_{2 n-1} & :=\tau_{n}
\end{aligned}
$$

for all positive integers $n$. We notice that for all $n \geq 1$

$$
\tau_{2^{n}+i}=1-\tau_{i} \text { for all } i=1, \ldots, 2^{n}
$$

Remark 15 Equality (8) characterises the Thue-Morse sequence via bitwise negation; indeed, it states that every initial block of length $2^{n}$, i.e, $\tau_{1}, \ldots, \tau_{2^{n}}$, is followed by a block of equal length that is its bitwise negation, i.e., $\tau_{2^{n}+1}=1-\tau_{1}, \ldots, \tau_{2^{n+1}}=$ $1-\tau_{2^{n}}$. This can be proved by an inductive argument, indeed the case $n=1$ follows by a direct computation and, assuming (8) as inductive hypothesis, one readily gets the inductive step

$$
\tau_{2^{n+1}+i}= \begin{cases}\tau_{2\left(2^{n}+i / 2\right)}=1-\tau_{2^{n}+i / 2}=1-\tau_{i / 2}=1-\tau_{i} & \text { if } i \text { is even } \\ \tau_{2\left(2^{n}+i / 2\right)}=\tau_{2^{n}+(i+1) / 2}=1-\tau_{(i+1) / 2}=1-\tau_{i} & \text { if } i \text { is odd }\end{cases}
$$

Lemma 16 For all $n \geq 2$

$$
L_{2^{n}}=-\underbrace{\sigma_{z} \otimes \cdots \otimes \sigma_{z}}_{n \text { times }}
$$

Moreover $L_{2^{n}}$ is a diagonal matrix whose diagonal elements $L_{2^{n}, i}, i=1, \ldots, 2^{n}$, satisfy

$$
L_{2^{n}, i}=2 \tau_{i}-1, \quad \text { for all } n=1, \ldots, 2^{n},
$$

where $\left(\tau_{i}\right)$ is the Thue-Morse sequence. 
Proof In order to prove (9), we recall the definition of $L_{2^{n}}$ in Eq. (7)

$$
\begin{aligned}
L_{2^{n}}= & B_{2^{n}}^{\dagger} M_{2^{n}} B_{2^{n}} \\
= & \left(L H_{2}\right)^{\dagger} \sigma_{y} R H_{2} \otimes \underbrace{\left(H_{2}^{\dagger} \sigma_{x} H_{2}\right) \otimes \cdots \otimes\left(H_{2}^{\dagger} \sigma_{x} H_{2}\right)}_{n-1 \text { times }} \otimes \sigma_{y} \sigma_{x}+ \\
& +\left(R H_{2}\right)^{\dagger} \sigma_{y} L H_{2} \otimes \underbrace{\left(\left(\sigma_{x} H_{2}\right)^{\dagger} H_{2}\right) \otimes \cdots \otimes\left(\left(\sigma_{x} H_{2}\right)^{\dagger} H_{2}\right)}_{n-1 \text { times }} \otimes \sigma_{x}^{\dagger} \sigma_{y}
\end{aligned}
$$

the second equality is obtained by applying Definition 11, Equations (5) and (6) where $B_{2^{n}}$ is given in terms of $\mathrm{CNOT}_{2^{n}}$, namely

$$
B_{2^{n}}=\left(L H_{2}\right) \otimes \underbrace{H_{2} \otimes \cdots \otimes H_{2}}_{(n-1) \text { times }}+\left(R H_{2}\right) \otimes \underbrace{\sigma_{x} H_{2} \otimes \cdots \otimes \sigma_{x} H_{2}}_{(n-1) \text { times }}
$$

and by applying $L^{\dagger} \sigma_{y} L=R^{\dagger} \sigma_{y} R=0$. By a direct computation

$\left(R H_{2}\right)^{\dagger} \sigma_{y} L H_{2}=\left(\left(L H_{2}\right)^{\dagger} \sigma_{y} R H_{2}\right)^{\dagger}=-\frac{i}{2}\left(\begin{array}{ll}-1 & 1 \\ -1 & 1\end{array}\right) \quad$ and $\quad \sigma_{x}^{\dagger} \sigma_{y}=\left(\sigma_{y} \sigma_{x}\right)^{\dagger}=i \sigma_{z}$.

By plugging above relations in (11), we obtain the first part of the claim, indeed

$$
\begin{aligned}
L_{2^{n}} & =\frac{i}{2}\left(\begin{array}{ll}
-1 & 1 \\
-1 & 1
\end{array}\right) \otimes \underbrace{\sigma_{z} \otimes \cdots \otimes \sigma_{z}}_{n-2 \text { times }} \otimes\left(-i \sigma_{z}\right)-\frac{i}{2}\left(\begin{array}{cc}
-1 & -1 \\
1 & 1
\end{array}\right) \otimes \underbrace{\sigma_{z} \otimes \cdots \otimes \sigma_{z}}_{n-2 \text { times }} \otimes i \sigma_{z} \\
& =\frac{1}{2}\left(\begin{array}{ll}
-1 & 1 \\
-1 & 1
\end{array}\right) \otimes \underbrace{\sigma_{z} \otimes \cdots \otimes \sigma_{z}}_{n-1 \text { times }}+\frac{1}{2}\left(\begin{array}{cc}
-1 & -1 \\
1 & 1
\end{array}\right) \otimes \underbrace{\sigma_{z} \otimes \cdots \otimes \sigma_{z}}_{n-1 \text { times }} \\
& =-\underbrace{\sigma_{z} \otimes \cdots \otimes \sigma_{z}}_{n \text { times }} .
\end{aligned}
$$

Now, above equality implies

$$
L_{2^{n}}=\sigma_{z} \otimes L_{2^{n-1}}
$$

and, by an inductive argument, that $L_{2^{n}}$ is a diagonal matrix.

Finally we prove (10) by induction on $n$. The base of induction, i.e. the case $n=1$, readily follows by $L_{2}=\sigma_{z}$ and by the definition of $\tau_{1}$ and of $\tau_{2}$. Now we prove the inductive step, i.e., we assume (10) as inductive hypothesis and we show

$$
L_{2^{n+1}, i}=2 \tau_{i}-1, \quad \text { for all } i=1, \ldots, 2^{n+1},
$$

By (12) we have $L_{2^{n+1}}=\sigma_{z} \otimes L_{2^{n}}$ and, consequently,

$$
L_{2^{n+1}, i}=\left\{\begin{array}{ll}
L_{2^{n}, i} & \text { if } i \leq 2^{n} \\
-L_{2^{n}, i-2^{n}} & \text { otherwise. }
\end{array} .\right.
$$


This, together with (8), implies (13), indeed we have

$$
\begin{aligned}
L_{2^{n+1}, i} & =L_{2^{n}, i}=2 \tau_{i}-1 \\
L_{2^{n+1}, 2^{n}+i} & =-L_{2^{n}, i}=1-2 \tau_{i}=2 \tau_{2^{n}+i}-1
\end{aligned}
$$

for all $i=1, \ldots, 2^{n}$ and this completes the proof.

Theorem 17 The $2^{n}$-dimensional Bell states are maximally entangled with respect to MW measure.

Proof By Lemma 16, $L_{2^{n}}$ is a diagonal matrix with 1 or -1 as diagonal elements then $\left|\left\langle k\left|L_{2^{n}}\right| \bar{k}\right\rangle\right|=1$ for all $k=0, \ldots, 2^{n}-1$ and this, together with Lemma 13, implies the claim.

\subsection{Some remarks on an entanglement criterion}

Lemma 13 provides a maximal entanglement criterion that can be rephrased as follows "If $\left|\left\langle\phi\left|L_{2^{n}}\right| \bar{\phi}\right\rangle\right|=1$ then $B_{2^{n}}|\phi\rangle$ is maximally entangled". Then one may ask how is made the space of states satisfying this condition. Lemma 16 provides some answers to this question. Indeed we already used in the proof of Theorem 17 the fact that $\left|\left\langle k\left|L_{2^{n}}\right| k\right\rangle\right|=1$, if $|k\rangle$ is an element of the canonical base. Next result investigates this property in the larger class of states whose coordinates in the standard base are real valued.

Proposition 18 Let $\left(\tau_{i}\right)$ be the Thue-Morse sequence and let $o_{i}$ and $e_{i}$ be the index sequences such that $\tau_{o_{i}}=1$ and $\tau_{e_{i}}=0$ for all $i \in \mathbb{N}$. Then for all $x \in \mathbb{R}^{2^{n}}$ with $|x|=1$, one has $\left|x^{\mathrm{T}} L_{2^{n}} x\right|=1$ if and only if either $x_{e_{i}}=0$ for all $i=1, \ldots, 2^{n-1}$ or $x_{o_{i}}=0$ for all $i=1, \ldots, 2^{n-1}$.

Proof Let $x=\left(x_{1}, \ldots, x_{2^{n}}\right) \in \mathbb{R}^{2^{n}}$ with $|x|=1$. Since $x$ is real valued then $\left|x_{i}\right|^{2}=x_{i}^{2}$ for all $i=1, \ldots, 2^{n}$ and $\sum_{i=1}^{2^{n}} x_{i}^{2}=|x|^{2}$. On the other hand $\left|x^{\mathrm{T}} L_{2^{n}} x\right|=$ $\left|\sum_{i=1}^{2^{n}} \tau_{i} x_{i}^{2}\right|=1$ if and only if either $\sum_{i=1}^{2^{n}} L_{2^{n}, i} x_{i}^{2}=1$ or $\sum_{i=1}^{2^{n}} L_{2^{n}, i} x_{i}^{2}=-1$, where $L_{2^{n}, i}$ is the $i$-th diagonal element of $L_{2^{n}}$. Since $|x|=1$, the former case is equivalent to $\sum_{i=1}^{2^{n}} L_{2^{n}, i} x_{i}^{2}=\sum_{i=1}^{2^{n}} x_{i}^{2}$ and, this, together with the equality $L_{2^{n}, i}=2 \tau_{i}-1$ proved in Lemma 16, implies

$$
0=\sum_{i=1}^{2^{n}}\left(L_{2^{n}, i}-1\right) x_{i}^{2}=\sum_{i=1}^{2^{n}}\left(2 \tau_{i}-2\right) x_{i}^{2}=-2 \sum_{i=1}^{2^{n-1}} x_{e_{i}}^{2}
$$

Above equality holds if and only if $x_{e_{i}}=0$ for all $i=1, \ldots, 2^{n-1}$. It follows by a similar argument that $\sum_{i=1}^{2^{n}} \tau_{i} x_{i}^{2}=-1$ is equivalent to $x_{o_{i}}=0$ for all $i=1, \ldots, 2^{n-1}$ and this completes the proof.

Remark 19 The matrix $L_{2^{n}}$ provides an explicit method for building maximally entangled states, indeed if $\left|\left\langle x^{\mathrm{T}} L_{2^{n}} x\right\rangle\right|=1$ then $B_{2^{n}}|x\rangle$ is a maximally entangled state. 
Lemma 16 points out the intimate relation between $L_{2^{n}}$ and the first $2^{n}$ terms of the Thue-Morse sequence $01101001 \cdots$. Indeed the $i$-th diagonal element of $L_{2^{n}}$ is $2 \tau_{i}-1$, that is, the diagonal elements of $L_{2 n}$ are the finite sequence of digits +1 and -1 obtained by replacing with -1 every occurence of 0 in the Thue-Morse sequence. Conway's evil and odious numbers are index sequences $e_{i}$ and $o_{i}$ associated with the Thue-Morse sequence by the following rule: $\tau_{e_{i}}=0$ for each $i \in \mathbb{N}$ and $\tau_{o_{i}}=1$ for each $i \in \mathbb{N}$. For instance, the first terms of $\left(e_{i}\right)$ are $1,4,6,7, \ldots$ while the first terms of complementary sequence $\left(o_{i}\right)$ are $2,3,5,8, \ldots$ Above Proposition characterises the set for each real valued unitary vectors $x \in \mathbb{R}^{n}$ satisfying $\left|x^{\mathrm{T}} L_{2^{n}} x\right|=1$ via a class of constraints on the occurrences the 0 's in $x$ based on evil and odious numbers. In particular it states that $x \in \mathbb{R}^{2^{n}}$ satisfies $\left|x^{\mathrm{T}} L_{2^{n}} x\right|=1$ if and only one of the following cases occurs: either $x$ belongs to the class of "evil" states (that is $x_{e_{i}}=0$ for each $i$ such that $e_{i} \leq 2^{n}$ ) or $x$ belongs to the class of "odious" states (that is $x_{d_{i}}=0$ for each $i$ such that $\left.e_{i} \leq 2^{n}\right)$. For instance $x=\frac{1}{2}(0,1,1,0,1,0,0,1)$ and $y=\frac{1}{\sqrt{2}}(0,1,0,0,0,0,0,0,1)$ are "evil" states, indeed $x_{1}=y_{1}=x_{4}=y_{4}=x_{6}=y_{6}=x_{7}=y_{7}=0$ (recall that the first evil numbers are 1, 4, 6, 7). It hence follows from Proposition 18 and Lemma 13 that $B_{2^{n}} x$ and $B_{2^{n}} y$ are maximally entangled states.

On the other hand, the state $z=\frac{1}{2}(1,1,1,1,0,0,0,0)$ is not "evil" nor "odious" because $z_{e_{1}} \neq 0$ and $z_{o_{1}} \neq 0$, therefore $B_{2^{n}} z$ may be not maximally entangled.

Finally note that Proposition 18 applies only to real valued vectors. For instance the state $z=\frac{1}{2}(1,0,0,1,0, i, i, 0)$ is "evil" and yet $\left|\left\langle x^{\mathrm{T}} L_{2^{n}} x\right\rangle\right|=0 \neq 1$; therefore, $B_{2^{n}}|z\rangle$ may be not a maximally entangled state.

\section{Conclusions}

We proposed a family of unitary transformations generalising the CNOT gate to an arbitrary number of qubits. We showed that a circuit composed by Walsh matrix and our general CNOT gate yields a maximally entangled (with respect to MW measure) set of states, that we called generalised Bell states. In order to prove the validity of the method, we developed ad hoc entanglement criteria based on the definition of a suitable antilinear operator. The paper also contains a preliminary theoretical investigation of such operator, which turned out to be related with the celebrated Thue-Morse sequence.

Results in the present paper open the way to further investigations in several directions. For instance, it could be interesting to extend the method to general controlled unitary operations. Also, a deeper investigation of antilinear operators with zero expectation value on product states could represent a step towards an algebraic characterisation of the states with maximal MW measure. Finally, it could be interesting to better understand the intriguing relation between states with maximal MW measure and the Thue-Morse sequence.

\section{References}

1. Ben-Aryeh Y.: The use of Braid operators for implementing entangled large $n$-qubits Bell states $(n>2)$. arXiv preprint arXiv:1403.2524, (2014) 
2. Bennett, C.H., Brassard, G., Crépeau, C., Jozsa, R., Peres, A., Wootters, W.K.: Teleporting an unknown quantum state via dual classical and Einstein-Podolsky-Rosen channels. Phys. Rev. Lett. 70(13), 18951899 (1993)

3. Bennett, C.H., DiVincenzo, D.P., Smolin, J.A., Wootters, W.K.: Mixed-state entanglement and quantum error correction. Phys. Rev. A 54(5), 3824 (1996)

4. Bennett, C.H., Popescu, S., Rohrlich, D., Smolin, J.A., Thapliyal, A.V.: Exact and asymptotic measures of multipartite pure-state entanglement. Phys. Rev. A 63(1), 012307 (2000)

5. Borras, A., Plastino, A.R., Batle, J., Zander, C., Casas, M., Plastino, A.: Multiqubit systems: highly entangled states and entanglement distribution. J. Phys. A: Math. Theor. 40(44), 13407 (2007)

6. Brennen, G.K.: An observable measure of entanglement for pure states of multi-qubit systems. Quantum Inf. Comput. 3(6), 619-626 (2003)

7. Brown, I.D.K., Stepney, S., Sudbery, A., Braunstein, S.L.: Searching for highly entangled multi-qubit states. J. Phys. A: Math. Gen. 38(5), 1119-1131 (2005). cited By 91

8. Eisert, J., Briegel, H.J.: Schmidt measure as a tool for quantifying multiparticle entanglement. Phys. Rev. A 64(2), 022306 (2001)

9. Ekert, A., Jozsa, R.: Quantum algorithms: entanglement-enhanced information processing. Philos. Trans. R. Soc. Lond Ser. A: Math. Phys. Eng. Sci. 356(1473), 1769-1781 (1998)

10. Gisin, N., Bechmann-Pasquinucci, H.: Bell inequality, Bell states and maximally entangled states for $n$ qubits. Phys. Lett. Sect. A: Gen. At. Solid State Phys. 246(1-2), 1-6 (1998). cited By 149

11. Graybill, F.A.: Matrices with Applications in Statistics. Wadsworth Statistics/Probability Series, 2nd edn., pp. xii+461. Wadsworth Advanced Books and Software, Belmont, CA (1983)

12. Grondalski, J.P., Etlinger, D.M., James, D.F.V.: The fully entangled fraction as an inclusive measure of entanglement applications. Phys. Lett. A 300(6), 573-580 (2002)

13. Heydari, H.: Topological quantum gate entanglers for a multi-qubit state. J. Phys. A: Math. Theor. 40(32), 9877 (2007)

14. Higuchi, A., Sudbery, A.: How entangled can two couples get? Phys. Lett. Sect. A: Gen. At. Solid State Phys. 273(4), 213-217 (2000). (cited By 68)

15. Jennewein, T., Simon, C., Weihs, G., Weinfurter, H., Zeilinger, A.: Quantum cryptography with entangled photons. Phys. Rev. Lett. 84(20), 4729 (2000)

16. Meyer, D.A., Wallach, N.R.: Global entanglement in multiparticle systems. J. Math. Phys. 43(9), 4273-4278 (2002). Quantum information theory

17. Osterloh, A., Siewert, J.: Constructing $n$-qubit entanglement monotones from antilinear operators. Phys. Rev. A 72, 012337 (2005)

18. Pathak, A.: Elements of Quantum Computation and Quantum Communication. Taylor \& Francis, Boca Raton, FL (2013)

19. Rieffel, E.G., Polak, W.H.: Quantum Computing: A Gentle Introduction. MIT Press, Cambridge, MA (2011)

20. Tapiador, J.E., Hernandez-Castro, J.C., Clark, J.A., Stepney, S.: Highly entangled multi-qubit states with simple algebraic structure. J. Phys. A: Math. Theor. 42(41), 415301 (2009)

21. Uhlmann, A.: Fidelity and concurrence of conjugated states. Phys. Rev. A 62, 032307 (2000)

22. Vidal, G.: Entanglement monotones. J. Mod. Opt. 47(2-3), 355-376 (2000)

23. Yang, C.P., Chu, S.I., Han, S.: Efficient many-party controlled teleportation of multiqubit quantum information via entanglement. Phys. Rev. A 70(2), 022329 (2004) 